\title{
Key Recovery und Vertrauen?
}

Bereits 1883 hat Auguste Kerckhoffs mit seinem Kerckhoffs'schen Prinzip einen Grundsatz der modernen Kryptographie formuliert. Danach begründet sich die Sicherheit eines Verschlüsselungsverfahrens nur auf der Geheimhaltung des Schlüssels, nicht aber auf der Geheimhaltung des verwendeten kryptographischen Algorithmus. Nahezu alle in Anwendung befindlichen, modernen kryptographischen Verfahren folgen diesem Prinzip - nicht zuletzt auch deshalb, weil die Vergangenheit gezeigt hat, dass dies der sicherste Weg ist. Nur in einigen wenigen Fällen wird noch die sogenannte „Security through obscurity“ angewendet: Sicherheit durch Geheimhaltung des (Verschlüsselungs-)Algorithmus oder auch Sicherheit durch eine geheim gehaltene Abwandlung eines öffentlich bekannten Algorithmus.

Seit die Kryptographie die Fesseln der Geheimdienste und des Militärs gesprengt hat und auch intensiv in Geschäfts- und Privatanwendungen benutzt wird, ist der Umgang mit dem „Schlüsselmaterial“ in den Fokus öffentlicher Diskussion gelangt. Zuerst wohl in Form der „Kryptokontroverse“, die in der zweiten Hälfte der 90er Jahre des 20. Jahrhunderts ihren Höhepunkt erlebte. Trivial formuliert ging es um die freie Verwendung - auch einer „starken“ - Kryptographie auf der einen Seite und den Versuch der Kontrolle über die Anwendungen durch „Herrschaft“ über die verwendeten Schlüssel auf der anderen Seite. Der Begriff Key Recovery tauchte erstmals im Zusammenhang mit der Exportkontrollpolitik der USA für starke Kryptographie auf. Kurz gesagt: Der Export von IT-Komponenten (in Hard- oder Software) mit sicherer Verschlüsselung (damals $>40$ Bit) sollte nur erlaubt sein, wenn die verwendeten Schlüssel entsprechenden staatlichen Stellen z. B. der NSA per Key Recovery verfügbar sind.

Eine Technologie, die bereits zu diesem Zeitpunkt das öffentliche Vertrauen in die kommerziell angebotenen Kryptographieanwendungen erschütterte, war Key Escrow. Dies meinte dabei hauptsächlich einen staatlicherseits erwünschten Einblick in und ggf. die Modifikation von kryptografische(n) Verschlüsselungsverfahren. Danach sollen entsprechende Schlüssel bei Dritten hinterlegt werden und staatliche Stellen unter gewissen Voraussetzungen Zugriff erhalten. Offiziell sind die Bemühungen, Key Escrow, gestützt auf aufwendige Entwicklungsprogramme in den USA, zu etablieren, Ende der 90er Jahre gescheitert. Die Sensibilität der Fachöffentlichkeit für das Interesse der „Dienste " an der Herrschaft über die kryptographischen Schlüssel ist jedoch nachhaltig stabilisiert worden. Wie berechtigt das Misstrauen ist, zeigt gerade jüngst der Vorfall rund um das PRISMSystem in den USA, das sich nach dem Stand der aktuellen Dis- kussion wohl in Datenbasen von US-Unternehmen mit globalen Nutzern bedienen kann.

Eine andere Herausforderung an das Vertrauen bei der Anwendung von Verschlüsselungsverfahren besteht aber gerade darin, dass der Verlust sicherer Schlüssel unweigerlich den Verlust der geschützten Information nach sich zieht. Das bedeutet letztendlich, dass jeder Besitzer von oder Verantwortliche für relevante Geheimnisse als Sicherheitsanker auch ein Backup des Schlüssels benötigt. Welche zumindest angemessen vertrauenswürdigen Lösungen dafür verfügbar sind, entscheidet oftmals, ob überhaupt durchgängig eine kryptographische Absicherung der Daten erzielbar ist.

Beteiligt an solchen Konzepten für ein Key Recovery können Implementierungen von Kryptographie in Hard- und Software, Rollen und Berechtigungen z. B. für Administratoren, sowie auch vertrauenswürdige externe Instanzen (z. B. Trustcenter) sein. Letztlich wird das Vertrauen in eine bestimmte Lösung aber immer durch subjektive Wahrnehmung - ggf. unterstützt durch Verträge und/oder Geschäftsbedingungen - bestimmt oder zumindest nachhaltig beeinflusst.

Große Unterschiede hinsichtlich der Erreichbarkeit von Schlüsselkopien bestehen zwischen Geschäftsprozessen und privaten Anwendungen:

In Unternehmen mit evaluierbaren IT-Sicherheitsregeln kann eine Sicherheitsarchitektur implementiert werden, die eine hinreichend sichere Ablage von Schlüsselkopien und die Schlüsselverfügbarkeit im Notfall - also etwa bei Verlust des Hauptschlüssels - gewährleistet.

Bei Privatanwendern sieht die Lage völlig anders aus, denn insbesondere durch die weitreichende Nutzung von Cloud-artigen Diensten steht der Privatanwender vor einem echten Dilemma: Entweder er vertraut niemandem und erleidet damit im Falle des Falles möglicherweise einen Datenverlust, weil auch die von ihm selbst sorgsam abgelegte Schlüsselkopie nicht mehr auffindbar ist. Oder er vertraut einem nicht kontrollierbaren Dritten, der zwar den Schlüssel im Notfall zur Verfügung stellen, aber bei dem eine missbräuchliche Verwendung - bspw. auch durch staatliche Stellen - niemals zweifelsfrei ausgeschlossen werden kann.

Letztendlich sind mit einem kryptographischen Verfahren geschützte Daten - entsprechende Sicherheit des verwendeten Verfahrens vorausgesetzt - immer nur so sicher wie der Schlüssel selbst. Sicher sind Daten aber übrigens auch, wenn der Schlüssel für niemanden mehr verfügbar ist - dann allerdings hat nicht nur der potentielle Angreifer ein Problem. 